\title{
Impact of Nonconcordance With NCCN Guidelines on Resource Utilization, Cost, and Mortality in De Novo Metastatic Breast Cancer
}

\author{
Gabrielle B. Rocque, MD, MSPH ${ }^{\mathrm{a}, \mathrm{b}}$; Courtney P. Williams, $\mathrm{MPH}^{\mathrm{b}}$; Bradford E. Jackson, $\mathrm{PhD}^{\mathrm{c}}$; \\ Stacey A. Ingram, MEd ${ }^{b}$ K Karian I. Halilova, MD, MPH' ; Maria Pisu, PhD, $;$ Kelly M. Kenzik, PhD, MS a,b, ; \\ Andres Azuero, PhD, MBA ${ }^{\mathrm{a}, \mathrm{f}}$; Andres Forero, MD ${ }^{\mathrm{a}, \mathrm{b}}$; and Smita Bhatia, MD, MPH ${ }^{\mathrm{a}, \mathrm{e}}$
}

\begin{abstract}
Background: The NCCN Clinical Practice Guidelines in Oncology (NCCN Guidelines) have directed the care of patients with cancer for $>20$ years. Payers are implementing guideline-based pathway programs that restrict reimbursement for non-guideline-based care to control costs, yet evidence regarding impact of guidelines on outcomes, including mortality, Medicare costs, and healthcare utilization, is limited. Patients and Methods: This analysis evaluated concordance of first treatment with NCCN Guidelines for women with de novo stage IV metastatic breast cancer (MBC) included within the SEER-Medicare linked database and diagnosed between 2007 and 2013. Cox proportional hazards models were used to evaluate the association between mortality and guideline concordance. Linear mixed-effects and generalized linear models were used to evaluate total cost to Medicare and rates of healthcare utilization by concordance status. Results: We found that $19 \%$ of patients (188/988) with de novo MBC received nonconcordant treatment. Patients receiving nonconcordant treatment were more likely to be younger and have hormone receptor-negative and HER2-positive MBC. The most common category of nonconcordant treatment was use of adjuvant regimens in the metastatic setting (40\%). Adjusted mortality risk was similar for patients receiving concordant and nonconcordant treatments (hazard ratio [HR], $0.85 ; 95 \%$ confidence limit $[\mathrm{CL}], 0.69,1.05$ ). When considering category of nonconcordance, patients receiving adjuvant regimens in the metastatic setting had a decreased risk of mortality $(\mathrm{HR}, 0.60 ; 95 \% \mathrm{CL}, 0.43$, 0.84). Nonconcordant treatments were associated with $\$ 1,867$ higher average Medicare costs per month compared with concordant treatments $(95 \% \mathrm{CL}, \$ 918, \$ 2,817)$. Single-agent HER2-targeted therapy was the highest costing category of nonconcordance at $\$ 3,008$ (95\% $\mathrm{CL}, \$ 1,014, \$ 5,001)$. Healthcare utilization rates were similar for patients receiving concordant and nonconcordant treatments. Conclusions: Despite a lack of survival benefit, concordant care was associated with lower costs, suggesting potential benefit to increasing standardization of care. These findings may influence policy decisions regarding implementation of pathway programs as health systems transition to value-based models.
\end{abstract}

aUniversity of Alabama at Birmingham Comprehensive Cancer Center, and

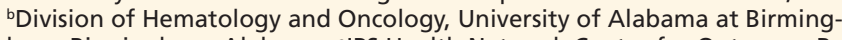
ham, Birmingham, Alabama; ‘JPS Health Network Center for Outcomes Research, Fort Worth, Texas; and dDivision of Preventive Medicine, eInstitute for Cancer Outcomes and Survivorship, and 'School of Nursing, University of Alabama at Birmingham, Birmingham, Alabama.

Submitted January 25, 2018; accepted for publication April 18, 2018.

Dr. Rocque has disclosed that she receives grant/research support Genentech, Pfizer, Carevive, PackHealth, and Medscape, and is a consultant for Pfizer. The remaining authors have disclosed that they have no financial interests, arrangements, affiliations, or commercial interests with the manufacturers of any products discussed in this article or their competitors.

Dr. Rocque received funding for this study from the Walter B. Frommeyer,
Jr. Fellowship in Investigative Medicine. This study used the linked SEERMedicare database. Interpretation and reporting of these data are the sole responsibility of the authors. The authors acknowledge the efforts of the $\mathrm{NCl}$; the Office of Research, Development and Information, CMS; Information Management Services (IMS), Inc.; and the SEER Program tumor registries in the creation of the SEER-Medicare database. Abstracts from this study were presented at the 2016 San Antonio Breast Cancer Symposium, December 6-10, 2016, and 2017 San Antonio Breast Cancer Symposium, December 5-9, 2017, San Antonio, Texas.

Correspondence: Gabrielle B. Rocque, MD, MSPH, Division of Hematology and Oncology, Department of Medicine, University of Alabama at Birmingham, WTI 240, 1720 2nd Avenue South, Birmingham, AL 35294. Email: grocque@uabmc.edu 
The NCCN Clinical Practice Guidelines in Oncology (NCCN Guidelines) have directed the care of patients with cancer for $>20$ years. ${ }^{1}$ In recent years, these guidelines have been further refined to include a category of "preferred" guideline treatment regimens to specify recommendations. ${ }^{2}$ Previous evaluations of NCCN Guidelines have focused on the use of specific, individual medications considered offlabel or nonconcordant. In breast cancer, $>10 \%$ of patients receive a medication not listed within the guidelines. ${ }^{3,4}$ These studies likely underestimate true levels of discordance because they do not capture concordance for regimens, which include specific single agents or combinations of hormone therapy, chemotherapy, and targeted therapies.

Use of nonconcordant treatments is known to contribute to the increasing cost of cancer care. ${ }^{5}$ The top 10 nonconcordant chemotherapy agents contribute to an estimated $\$ 2.5$ billion in US cancer spending. ${ }^{6}$ Patient factors are known to contribute to nonconcordant care, ${ }^{4}$ but the role of provider factors is less clear. In an effort to minimize provider-based treatment variability, payers and health systems are implementing pathway programs restricting reimbursement for off-pathway regimens. ${ }^{7-10}$ Although these programs are showing early promise, few data exist about the outcomes for nonconcordant treatment regimens, particularly in metastatic breast cancer $(\mathrm{MBC})$, in which treatment is heterogeneous and complex due to the large number of available guideline-based options. To ensure optimal patient care, it is essential to understand the types of commonly prescribed nonconcordant treatments and their impact on mortality, total Medicare costs, and rates of healthcare utilization.

The objectives of this study were to examine regimens concordant with NCCN Guidelines for $\mathrm{MBC}$; describe categories of nonconcordant treatments; evaluate patient- and provider-level factors associated with nonconcordance; and determine the impact of nonconcordance on mortality, Medicare spending, and rates of healthcare utilization.

\section{Patients and Methods}

\section{Data Sources}

The SEER-Medicare linked database was used to evaluate concordance between NCCN Guidelines and MBC treatments from 2007 to 2013. ${ }^{11}$

\section{Patient Population}

This analysis included patients with de novo stage IV disease diagnosed between 2007 and 2013 who received treatment with chemotherapy, hormone therapy, or targeted therapy (see supplemental eFigure 1, available with article at JNCCN.org). The SEER-Medicare linked database included Medicare claims data from January 1, 2007, to December 31, 2013; patients without Medicare Parts A, B, and D coverage at the time of treatment were excluded. Other exclusion criteria included male sex and patients with probable billing errors as identified by simultaneous receipt of same-class agents (eg, paclitaxel/docetaxel, carboplatin/cisplatin, doxorubicin/ pegylated doxorubicin). All claims after enrollment in an $\mathrm{HMO}$ were excluded.

\section{Treatment Regimens and Guideline Concordance}

Characterizing Treatment Regimens: Hormonal medications, chemotherapy, and HER2-targeted therapy were identified from Medicare claims using National Drug Codes (NDCs), the Healthcare Common Procedure Coding System (HCPCS), and generic drug names. If multiple drug claims were billed on the same day, the treatments were classified as given jointly. The initial regimen (either single drug or combination) was identified as the first claim for treatment after the diagnosis date. Nonconcordant treatments were reviewed individually and grouped into discrete categories.

Concordance With NCCN Guidelines: A regimen was considered guideline-concordant if the treatment received corresponded to a treatment listed on the version of NCCN Guidelines published at the time of treatment. Concordant regimens were further categorized as "preferred" or "other." Due to separate NCCN algorithms for treatment, chemotherapy concordance for HER2-positive and HER2-negative breast cancers was evaluated independently. Patients with unknown HER2 status were considered concordant if they received either a HER2-positive or HER2-negative regimen.

\section{Outcomes}

Survival: Survival was assessed as time from first treatment until death (all-cause and breast cancerspecific) or censoring by both concordance status overall and by type of nonconcordant treatment. 
Rocque et al

Costs to Medicare and Healthcare Utilization: Costs to Medicare were assessed using reimbursements to providers from the inpatient (Medicare Provider Analysis and Review [MEDPAR]), outpatient (Outpatient Standard Analytic File), National Claims History, and durable medical equipment files. Costs were summed and averaged per month based on available patient follow-up months. Part D costs were excluded because of the inability to differentiate costs to Medicare versus costs to patients and other payers. Costs to Medicare and healthcare utilization, defined as emergency department visits or inpatient hospitalizations, were identified from the start of cancer treatment until death or censoring (defined as last available claim per patient). Date of last follow-up was December 31, 2013.

\section{Covariates}

Patient Characteristics: The following patient characteristics were evaluated: age at diagnosis, race (white vs black vs other), census track poverty level ( $<20 \%$ federal poverty level vs $\geq 20 \%$ poverty), census track education level (mean \% non-high school education vs mean \% high school education or greater), dual eligibility (ever vs never), residence (urban vs rural), Medicare reason for entitlement (age vs disability/end-stage renal disease), year of treatment, hormone receptor status (positive vs negative vs unknown), and HER2 status (positive vs negative vs unknown). Comorbid conditions in the year prior to diagnosis were abstracted from claims data and classified using a weighted Charlson comorbidity index score of $0,1,2$ to 3 , or $\geq 4$ based on the Klabunde modification for comorbidities. ${ }^{12-14}$

Prescribing Providers: National Provider Identifiers (NPIs) or Unique Physician Identification Numbers (UPINs) were used to designate prescribing providers, defined as the oncology provider directly linked to the treatment claim. For patients receiving oral treatment, no linked provider existed. Therefore, we identified the oncology provider with the closest evaluation and management $(E / M)$ code on or prior to the first treatment date. The closest nononcology provider was designated as the prescribing provider if no oncology-specific provider was identified using $\mathrm{E} / \mathrm{M}$ codes. Each prescribing provider was then assigned a hospital based on the practice location associated with the highest number of provider-spe- cific inpatient and outpatient claims. The following provider characteristics were abstracted from the American Medical Association (AMA) database: sex (male vs female), years since medical school graduation, medical training location (United States vs foreign), and primary and secondary specialty (oncology vs other). Additional provider characteristics were identified based on their affiliated hospital and obtained from the American Hospital Association database: SEER region (Northeast vs South vs Midwest vs West), medical school or NCI designation (medical school and NCI designation vs medical school and no NCI designation vs neither), rural hospital status (rural primary hospital qualification or rural hospital location or critical access hospital vs none), total number of beds, and cooperative group count. The number of cooperative groups was defined as the number of groups that the institution is enrolled in with common clinical trial protocols.

\section{Statistical Analysis}

Summary data consisted of means and standard deviations for continuous variables or frequencies and percentages for categorical variables. Risk ratios (RRs) for patient- and provider-level factors associated with nonconcordance were calculated using generalized log-linear models with a Poisson distribution and robust variance estimates. All-cause and breast cancerspecific mortality between patients on concordant versus nonconcordant treatments were compared using Cox proportional hazards models. Models were adjusted for hormone receptor/HER2 status, Charlson comorbidity score, age at diagnosis, race, and census tract poverty to account for confounding control. Proportionality of the hazard assumption was assessed using the weighted Schoenfeld residual method by Grambsch and Therneau. ${ }^{15}$ To evaluate the association of healthcare utilization and nonconcordant treatment, RRs and 95\% confidence limits (CLs) were estimated using generalized linear models with a binomial or Poisson distribution and log link function. The log of total follow-up months was used as an offset in the Poisson models. To evaluate the impact of nonconcordance on cost, linear mixed-effects models were used to evaluate trends in average monthly cost. Utilization and cost models were adjusted for calendar time to account for policy changes that could affect either outcome over time; utilization models were also adjusted for length of patient follow-up to account for 
patient-time allowed for events. Sensitivity analyses were performed to examine potential differences in concordance by presence of known versus unknown receptor statuses and for treatment received by oncologist versus other provider. All analyses were performed using SAS 9.4 (SAS Institute Inc.). This study was approved by University of Alabama at Birmingham's Institutional Review Board.

\section{Results}

\section{Patient Population}

A total of 988 patients received treatment for de novo MBC between 2007 and 2013 (eFigure 1). The analysis included 26,187 person-months of followup, and median follow-up was 2.1 years (interquartile range, 3.0 years). Demographic characteristics

\section{Table 1. Patient Demographics ( $N=988)$}

\begin{tabular}{|c|c|c|c|}
\hline & \multicolumn{3}{|c|}{ De Novo Stage IV } \\
\hline & $\begin{array}{l}\text { Concordant } \\
(\mathrm{N}=800)\end{array}$ & $\begin{array}{l}\text { Nonconcordant } \\
\quad(\mathrm{N}=188)\end{array}$ & $P$ Value \\
\hline $\begin{array}{l}\text { Mean age at first treatment } \\
\text { (SD), y }\end{array}$ & $73.7(10.0)$ & $70.2(8.6)$ & $<.001$ \\
\hline Race, n (\%) & & & .66 \\
\hline White & $636(79.5 \%)$ & $144(76.6 \%)$ & \\
\hline Black & $104(13.0 \%)$ & $27(14.4 \%)$ & \\
\hline Other & $60(7.5 \%)$ & $17(9.0 \%)$ & \\
\hline Residence, n (\%) & & & .27 \\
\hline Rural & $107(13.4 \%)$ & $31(16.5 \%)$ & \\
\hline Urban & $693(86.6 \%)$ & $157(83.5 \%)$ & \\
\hline Census tract poverty, $\mathrm{n}(\%)$ & & & .16 \\
\hline$<20 \%$ poverty & $598(74.8 \%)$ & $131(69.7 \%)$ & \\
\hline$\geq 20 \%$ poverty & $202(25.3 \%)$ & $57(30.3 \%)$ & \\
\hline Reason for entitlement, n (\%) & & & .01 \\
\hline Age $\geq 65$ y & $692(86.5 \%)$ & $149(79.3 \%)$ & \\
\hline Disability/ESRD & $108(13.5 \%)$ & $39(20.7 \%)$ & \\
\hline \multicolumn{2}{|l|}{ Ever dual eligible (Medicaid), n (\%) } & & .53 \\
\hline Yes & $316(39.5 \%)$ & $79(42.0 \%)$ & \\
\hline No & $484(60.5 \%)$ & $109(58.0 \%)$ & \\
\hline \multicolumn{4}{|c|}{ Mean $\%$ census tract education level (SD) ${ }^{a}$} \\
\hline Non-high school education & $21.4(14.1)$ & $23.2(15.7)$ & .13 \\
\hline $\begin{array}{l}\text { High school education or } \\
\text { greater }\end{array}$ & $78.6(14.1)$ & $76.8(15.7)$ & .12 \\
\hline Year of treatment, ${ }^{b} \mathrm{n}(\%)$ & & & .02 \\
\hline 2007 & $163(20.4 \%)$ & $20(10.6 \%)$ & \\
\hline 2008 & $144(18.0 \%)$ & $37(19.7 \%)$ & \\
\hline 2009 & $148(18.5 \%)$ & $31(16.5 \%)$ & \\
\hline 2010 & $160(20.0 \%)$ & $41(21.8 \%)$ & \\
\hline 2011 & $167(20.9 \%)$ & $54(28.7 \%)$ & \\
\hline Hormone receptor status, $\mathrm{n}(\%)$ & & & $<.001$ \\
\hline Positive & $614(76.8 \%)$ & $94(50.0 \%)$ & \\
\hline Negative & $92(11.5 \%)$ & $78(41.5 \%)$ & \\
\hline Unknown & $94(11.8 \%)$ & $16(8.5 \%)$ & \\
\hline HER2 status, $\mathrm{n}(\%)$ & & & $<.001$ \\
\hline Positive & $42(5.3 \%)$ & $54(28.7 \%)$ & \\
\hline Negative & $235(29.4 \%)$ & $32(17.0 \%)$ & \\
\hline Unknown & $523(65.4 \%)$ & $102(54.3 \%)$ & \\
\hline
\end{tabular}

Abbreviation: ESRD, end-stage renal disease.

${ }^{\mathrm{a}} \mathrm{N}=987$

b2012-2013 excluded due to insufficient sample size. are shown in Table 1 . Patients receiving nonconcordant treatment were younger and had hormone receptor-negative and HER2-positive disease.

\section{Concordance With NCCN Guidelines}

A total of $81 \%$ of patients received guideline concordant initial treatment, with $80 \%$ receiving a "preferred" regimen and $<1 \%$ receiving an "other" on-guideline regimen. Sensitivity analyses excluding unknown receptor status (ie, HR and HER2) showed no difference in concordance status. The following categories of nonconcordant treatment $(n=188)$ were identified: (1) single-agent HER2-targeted therapy (19\%), (2) adjuvant regimens used in the metastatic setting (40\%), (3) nonapproved bevacizumab regimens $(6 \%),(4)$ therapy mismatched with estrogen receptor (ER)/HER2 status (16\%), and (5) other miscellaneous reasons (19\%). The most common reason for nonconcordant treatment was use of adjuvant regimens (40\%; Figure 1), which included docetaxel/cyclophosphamide (TC) and docetaxel/ doxorubicin/cyclophosphamide (TAC). Within the nonapproved bevacizumab category, bevacizumab was used in 10 different drug combinations.

Factors Associated With Nonconcordance: Patients more likely to receive nonconcordant treatment were younger and had hormone receptor-negative and HER2-positive disease (supplemental eTable 1). Considering provider characteristics, increasing years from training, oncology specialty, and decreasing number of cooperative group involvement

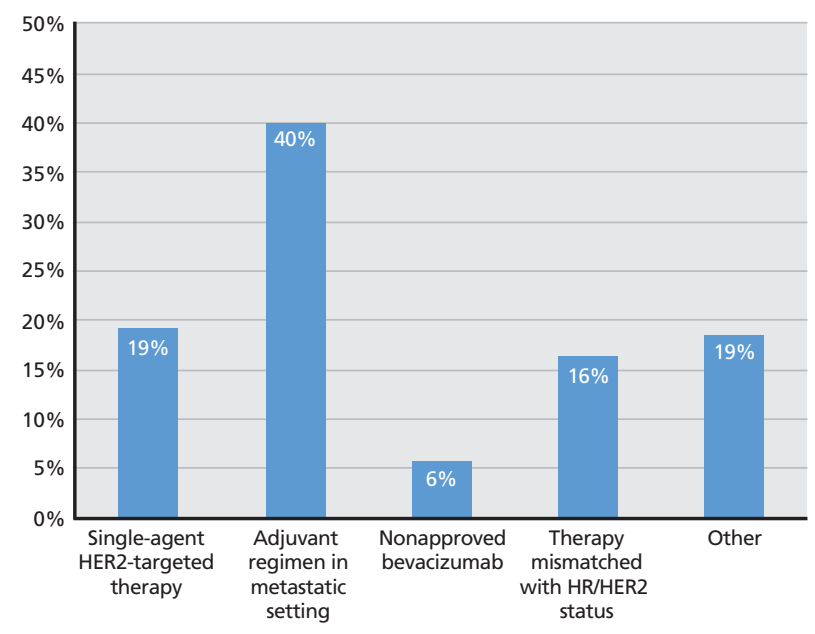

Figure 1. Reasons for nonconcordance in de novo stage IV metastatic breast cancer $(n=188)$.

Abbreviation: HR, hormone receptor. 
increased the likelihood of nonconcordant treatments (supplemental eTable 1). Sensitivity analyses showed similar results after excluding nononcology providers (11\% of all included providers).

Mortality: Adjusted all-cause mortality risk was slightly lower for patients receiving concordant versus nonconcordant treatments (hazard ratio [HR], 0.85; 95\% CL, 0.69, 1.05; Figure 2A). After considering type of nonconcordant treatment, use of adjuvant regimens in the metastatic setting was associated with a decreased risk of all-cause mortality (HR, 0.60; 95\% CL, 0.43, 0.84; Figure 2B; Table 2). Breast cancer-specific mortality did not differ by concordance status overall or by type of nonconcordant treatment (Table 2).

Costs to Medicare and Healthcare Utilization: Nonconcordant treatments were associated with $\$ 1,867$ higher average Medicare costs per month compared with concordant treatments $(95 \% \mathrm{CL}, \$ 918, \$ 2,817$;
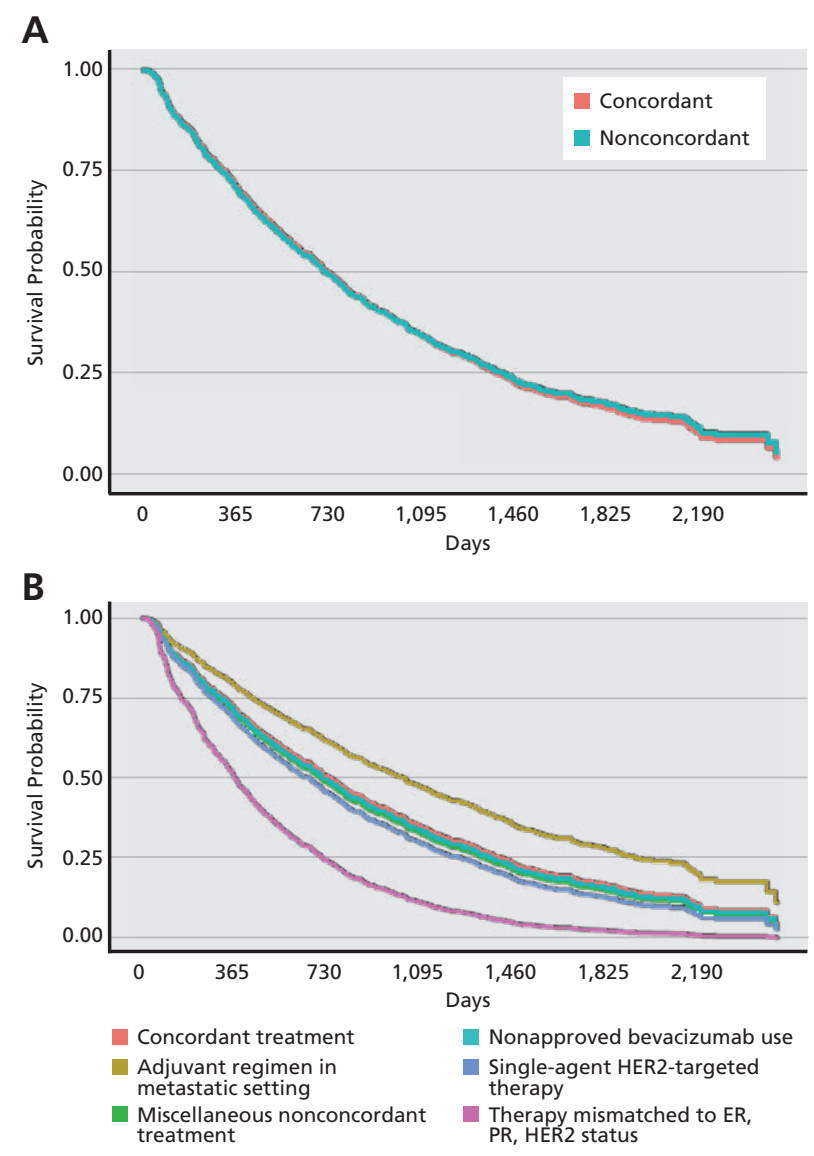

Figure 2. Survivor function curves for (A) overall survival and (B) overall survival stratified by concordance category in de novo stage IV metastatic breast cancer $(\mathrm{N}=988)$.

Abbreviations: ER, estrogen receptor; PR, progesterone receptor.

\begin{tabular}{|c|c|c|c|}
\hline & $\begin{array}{c}\text { Hazard } \\
\text { Ratio }\end{array}$ & $\begin{array}{c}\text { Lower } \\
\text { CL }\end{array}$ & $\begin{array}{c}\text { Upper } \\
\text { CL }\end{array}$ \\
\hline \multicolumn{4}{|l|}{ All-cause unadjusted model } \\
\hline Concordant & Ref & - & - \\
\hline $\begin{array}{l}\text { Adjuvant regimen in metastatic } \\
\text { setting }\end{array}$ & 0.61 & 0.44 & 0.85 \\
\hline Nonapproved bevacizumab use & 1.03 & 0.53 & 1.99 \\
\hline Other & 1.59 & 1.11 & 2.28 \\
\hline $\begin{array}{l}\text { Single-agent HER2-targeted } \\
\text { therapy }\end{array}$ & 1.16 & 0.80 & 1.67 \\
\hline $\begin{array}{l}\text { Therapy not matched with } \\
\text { hormone/HER2 receptor status }\end{array}$ & 1.40 & 0.92 & 2.15 \\
\hline \multicolumn{4}{|l|}{ All-cause adjusted modela } \\
\hline Concordant & Ref & - & - \\
\hline $\begin{array}{l}\text { Adjuvant regimen in metastatic } \\
\text { setting }\end{array}$ & 0.60 & 0.43 & 0.84 \\
\hline Nonapproved bevacizumab use & 0.82 & 0.42 & 1.60 \\
\hline Other & 1.38 & 0.95 & 1.99 \\
\hline $\begin{array}{l}\text { Single-agent HER2-targeted } \\
\text { therapy }\end{array}$ & 0.92 & 0.62 & 1.35 \\
\hline $\begin{array}{l}\text { Therapy not matched with } \\
\text { hormone/HER2 receptor status }\end{array}$ & 1.10 & 0.68 & 1.78 \\
\hline \multicolumn{4}{|c|}{ Breast cancer-specific unadjusted model } \\
\hline Concordant & Ref & - & - \\
\hline $\begin{array}{l}\text { Adjuvant regimen in metastatic } \\
\text { setting }\end{array}$ & 0.70 & 0.46 & 1.07 \\
\hline Nonapproved bevacizumab use & 1.15 & 0.55 & 2.43 \\
\hline Other & 1.11 & 0.80 & 1.55 \\
\hline $\begin{array}{l}\text { Single-agent HER2-targeted } \\
\text { therapy }\end{array}$ & 0.99 & 0.62 & 1.59 \\
\hline $\begin{array}{l}\text { Therapy not matched with } \\
\text { hormone/HER2 receptor status }\end{array}$ & 2.11 & 0.94 & 4.71 \\
\hline \multicolumn{4}{|l|}{ Breast cancer-specific adjusted model ${ }^{\mathrm{a}}$} \\
\hline Concordant & Ref & - & - \\
\hline $\begin{array}{l}\text { Adjuvant regimen in metastatic } \\
\text { setting }\end{array}$ & 0.71 & 0.46 & 1.09 \\
\hline Nonapproved bevacizumab use & 0.92 & 0.43 & 1.98 \\
\hline Other & 1.13 & 0.79 & 1.61 \\
\hline $\begin{array}{l}\text { Single-agent HER2-targeted } \\
\text { therapy }\end{array}$ & 0.84 & 0.52 & 1.37 \\
\hline $\begin{array}{l}\text { Therapy not matched with } \\
\text { hormone/HER2 receptor status }\end{array}$ & 0.99 & 0.43 & 2.27 \\
\hline
\end{tabular}

Abbreviation: $\mathrm{CL}$, 95\% confidence limit.

${ }^{a}$ Adjusted models included hormone receptor status, HER2 status, Charlson

comorbidity score, age at diagnosis, race, and census track poverty.

Table 3). When considering the category of nonconcordance, single-agent HER2-targeted therapy was associated with the highest average monthly cost to Medicare compared with concordant treatment $(\beta$, $\$ 3,008 ; 95 \% \mathrm{CL}, \$ 1,014, \$ 5,001)$. Number of emergency department visits and hospitalizations did not differ between patients receiving concordant versus nonconcordant treatment (RR, 1.11; 95\% CL, 0.86, 1.44 , and $R R, 1.10 ; 95 \%$ CL, 0.94, 1.29 , respectively).

\section{Discussion}

To our knowledge, this is the first analysis of the impact of nonconcordant treatment type on mortality and healthcare utilization in de novo MBC. We observed that almost 1 in 5 patients received nonconcordant 
NCCN Guideline Nonconcordance

\begin{tabular}{|c|c|c|c|c|}
\hline Costs to Medicare $^{a}$ & $\beta^{\mathrm{b}}$ & Lower CL & Upper CL & $P$ Value \\
\hline Average monthly cost (\$US) & 1,867 & 918 & 2,817 & $<.001$ \\
\hline Adjuvant regimen in metastatic setting & 1,237 & -184 & 2,658 & .09 \\
\hline Nonapproved bevacizumab use & 2,588 & -965 & 6,140 & .15 \\
\hline Single-agent HER2-targeted therapy & 3,008 & 1,014 & 5,001 & .003 \\
\hline Therapy mismatched with hormone/HER2 receptor status & 2,330 & 187 & 4,474 & .03 \\
\hline Healthcare Utilization ${ }^{c}$ & $\mathbf{R R}$ & Lower $\mathrm{CL}$ & Upper CL & $P$ Value \\
\hline Any ED visit & 1.11 & 0.79 & 1.56 & .55 \\
\hline Adjuvant regimen in metastatic setting & 1.26 & 0.75 & 2.14 & .39 \\
\hline Nonapproved bevacizumab use & 1.33 & 0.39 & 4.50 & .65 \\
\hline Single-agent HER2-targeted therapy & 0.86 & 0.43 & 1.71 & .67 \\
\hline Therapy mismatched with hormone/HER2 receptor status & 0.62 & 0.28 & 1.38 & .24 \\
\hline Any hospitalization & 1.46 & 0.91 & 2.34 & .10 \\
\hline Adjuvant regimen in metastatic setting & 1.26 & 0.65 & 2.46 & .50 \\
\hline Nonapproved bevacizumab use & 1.70 & 0.22 & 12.90 & .61 \\
\hline Single-agent HER2-targeted therapy & 1.10 & 0.43 & 2.79 & .85 \\
\hline Therapy mismatched with hormone/HER2 receptor status & 1.52 & 0.51 & 4.52 & .45 \\
\hline Number of ED visits & 1.11 & 0.86 & 1.44 & .44 \\
\hline Adjuvant regimen in metastatic setting & 1.20 & 0.82 & 1.73 & .35 \\
\hline Nonapproved bevacizumab use & 0.94 & 0.48 & 1.87 & .86 \\
\hline Single-agent HER2-targeted therapy & 1.15 & 0.69 & 1.91 & .59 \\
\hline Therapy mismatched with hormone/HER2 receptor status & 0.58 & 0.31 & 1.08 & .08 \\
\hline Number of hospitalizations & 1.10 & 0.94 & 1.29 & .23 \\
\hline Adjuvant regimen in metastatic setting & 1.05 & 0.82 & 1.34 & .71 \\
\hline Nonapproved bevacizumab use & 1.03 & 0.66 & 1.60 & .90 \\
\hline Single-agent HER2-targeted therapy & 1.35 & 0.97 & 1.87 & .08 \\
\hline Therapy mismatched with hormone/HER2 receptor status & 1.02 & 0.72 & 1.44 & .91 \\
\hline
\end{tabular}

Abbreviations: $C L, 95 \%$ confidence limit; $E D$, emergency department; RR, risk ratio.

${ }^{a} G$ Guideline nonconcordant vs guideline concordant; model adjusted for calendar time.

${ }^{b}$ Cost difference between nonconcordant vs concordant treatment.

'Guideline nonconcordant vs guideline concordant; model adjusted for calendar time and length of patient follow-up.

first-line care. This is a higher proportion than reported in previous studies focusing on single medications, ${ }^{3,4}$ in which authors may have underestimated the use of standard therapy in combinations not included in guidelines. This is supported by our analysis of nonconcordance categories, which primarily included traditional breast cancer medications used within combinations inconsistent with NCCN Guidelines. These deviations may be considered modest compared with using off-label medications not approved for use in breast cancer. However, it is important to recognize that these approaches are not standard of care and do not carry the weight of evidence required to be included in the NCCN Guidelines.

In early-stage breast cancer, receipt of guidelinebased care is associated with better all-cause survival. ${ }^{16-18}$ Although a trend was seen toward a survival benefit, we found no statistically significant difference for patients receiving concordant versus nonconcordant treatment. This lack of difference may be due to the heterogeneity of care, as suggested by the varying outcomes observed by category of nonconcordance. In fact, the most common type of nonconcordant treatment-use of adjuvant regimens (TC and TAC) in the metastatic setting - was associated with a survival advantage. The recommendation for sequential, sin- gle-agent therapy over chemotherapy combinations used in the adjuvant setting has evolved over time and has been controversial. Three Cochrane reviews have been conducted with varying recommendations from 2005 to 2013, with the current recommendation against combination chemotherapy outside of the need for a rapid clinical response. ${ }^{19-21}$ However, these reviews do not consider patients with de novo $\mathrm{MBC}$ separately from those with recurrent MBC. This analysis suggests that there may be a subset of patients with de novo MBC who have not received prior chemotherapy and may benefit from the more aggressive, up-front combination therapy commonly prescribed in the adjuvant setting. For example, patients with low-volume or oligometastatic disease may benefit from more aggressive medical treatment, given that prior research suggests that some patients receive benefit from surgery or radiation for oligometastatic disease. ${ }^{22}$ However, NCCN Guidelines do not currently provide alternate recommendations for more aggressive treatment in these patients. Further work is needed to specifically address which patients could benefit from more aggressive approaches.

Both patient and provider factors influenced likelihood of receiving concordant care. Increased years 
Rocque et al

since provider training, oncology provider specialty, and hospital participation in fewer cooperative groups was associated with guideline nonconcordance in MBC. This large, nationwide study $(\mathrm{N}=988)$ is consistent with a prior smaller study $(\mathrm{N}=107)$ by Denu et $\mathrm{al}^{18}$ in inflammatory breast cancer, which found lower guideline concordance for physicians $>15$ years from medical school graduation and practicing at smaller hospitals. Younger physicians and individuals routinely participating in clinical trials as part of cooperative groups may have greater familiarity with recent guideline updates. This may be particularly relevant for the nonconcordant category of adjuvant therapy in the metastatic setting, given that the recommendation for sequential therapy rather than combination therapy is based on a meta-analysis performed in 2013. ${ }^{21}$ Thus, physicians may have been trained to use different approaches in this population based on when their training occurred. Other factors may have contributed to the observed differences in likelihood of concordant care that SEER-Medicare and AMA data do not capture, such as provider belief in the importance of guideline-based care or patient preferences.

This study also highlights one of the limitations of NCCN Guidelines, which are overly inclusive in nature. Despite the many available options within the NCCN Guidelines, $<1 \%$ of patients received a regimen that was considered on-guideline but was not on the "preferred" list. NCCN has made substantial improvements in the usability of the guidelines by adding evidence blocks that include comparisons between therapies regarding efficacy, safety, quality of evidence, consistency of evidence, and affordability of regimen/agent, although these were not available when this study was conducted. ${ }^{23}$ These evidence blocks provide a mechanism for providers to personalize treatment options.

Higher rates of healthcare utilization and higher Medicare costs were demonstrated in this study for patients receiving nonconcordant care, supporting ongoing efforts at treatment standardization as a method to reduce healthcare costs. These findings are consistent with those of previously reported studies on pathway programs that limit off-pathway treatment. ${ }^{7,9,10,24,25}$ At the same time, guideline concordance should rarely, if ever, be $100 \% .{ }^{10}$ The art of medicine includes the ability of physicians to tailor therapy to patient preferences and comorbidities and clinical scenario; pathways and data-driven approaches should never replace physician judgement.
This study provides valuable insights into the treatment of de novo MBC, but use of SEER-Medicare claims data has limitations. This study includes only patients with fee-for-service Medicare plans, which may not be representative of all patients with $\mathrm{MBC}$, including those who are younger and have commercial insurance. ${ }^{11}$ Older Medicare patients are likely to have hormone receptor-positive disease treated with hormone therapy, which is both well tolerated and guideline-concordant. In contrast, younger patients with commercial insurance are more likely to have hormone receptornegative disease and may receive aggressive, potentially discordant treatments. Although comorbidities were similar between groups, lower functional status or specific medical conditions may drive nonconcordant care toward less toxic regimens, which may explain the use of single-agent HER2-targeted therapy (19\%). However, our data suggest that escalation of care was more common than selection of a less toxic regimen, with $46 \%$ of patients receiving either a nonapproved combination chemotherapy or a bevacizumab-containing regimen. Finally, the lack of HER2 status in SEERMedicare data until 2010 is a limitation, which may result in overestimation of concordance because HER2unknown patients were considered concordant if they received treatment for either HER2-positive or HER2negative $\mathrm{MBC}$. This may contribute to the higher cost for trastuzumab alone, if patients with HER2-positive disease were treated with hormone therapy alone rather than combination chemotherapy. However, sensitivity analyses showed similar rates of guideline concordance after excluding patients with unknown HER2 status.

\section{Conclusions}

First-line treatment inconsistent with NCCN Guidelines was common, with $>19 \%$ of patients with $\mathrm{MBC}$ receiving nonconcordant initial cancer treatment. Healthcare utilization and Medicare costs were higher for patients receiving nonconcordant treatments. Although mortality was not substantially higher among these patients, mortality risk varied (in both directions) depending on the specific nonconcordant regimen. Although increasing standardization presents an opportunity to enhance healthcare value, further research is needed to understand the rationale for and outcomes associated with nonconcordant care. 


\section{References}

1. NCCN. Development and Update of the NCCN Guidelines. Available at: http://www.nccn.org/professionals/development.aspx. Accessed May $11,2015$.

2. Gradishar WJ, Anderson BO, Balassanian R, et al. Breast cancer version 2.2015. J Natl Compr Canc Netw 2015;13:448-475.

3. Eaton AA, Sima CS, Panageas KS. Prevalence and safety of off-label use of chemotherapeutic agents in older patients with breast cancer: estimates from SEER-Medicare data. J Natl Compr Canc Netw 2016;14:57-65.

4. Hamel S, McNair DS, Birkett NJ, et al. Off-label use of cancer therapies in women diagnosed with breast cancer in the United States. Springerplus 2015;4:209.

5. Shih YC, Ganz PA, Aberle D, et al. Delivering high-quality and affordable care throughout the cancer care continuum. J Clin Oncol 2013;31:41514157.

6. Conti RM, Bernstein AC, Villaflor VM, et al. Prevalence of off-label use and spending in 2010 among patent-protected chemotherapies in a populationbased cohort of medical oncologists. J Clin Oncol 2013;31:1134-1139.

7. Zon RT, Frame JN, Neuss MN, et al. American Society of Clinical Oncology policy statement on clinical pathways in oncology. J Oncol Pract 2016;12:261-266

8. Neubauer MA, Hoverman JR, Kolodziej M, et al. Cost effectiveness of evidence-based treatment guidelines for the treatment of non-small-cell lung cancer in the community setting. J Oncol Pract 2010;6:12-18.

9. Hoverman JR, Cartwright TH, Patt DA, et al. Pathways, outcomes, and costs in colon cancer: retrospective evaluations in 2 distinct databases. Am J Manag Care 2011;17(Suppl 5):SP45-52.

10. Polite BN, Page RD, Nabhan C. Oncology pathways-preventing a good idea from going bad. JAMA Oncol 2016;2:297-298.

11. Ambs A, Warren JL, Bellizzi KM, et al. Overview of the SEER-Medicare Health Outcomes Survey linked dataset. Health Care Financ Rev 2008;29:5-21.

12. Klabunde CN, Potosky AL, Legler JM, Warren JL. Development of a comorbidity index using physician claims data. J Clin Epidemiol 2000;53:1258-1267.
13. Charlson ME, Pompei P, Ales KL, MacKenzie CR. A new method of classifying prognostic comorbidity in longitudinal studies: development and validation. J Chronic Dis 1987;40:373-383.

14. Deyo RA, Cherkin DC, Ciol MA. Adapting a clinical comorbidity index for use with ICD-9-CM administrative databases. J Clin Epidemiol 1992;45:613-619.

15. Grambsch PM, Therneau TM. Proportional hazards tests and diagnostics based on weighted residuals. Biometrika 1994;81:515-526.

16. Schwentner L, Wockel A, Konig J, et al. Adherence to treatment guidelines and survival in triple-negative breast cancer: a retrospective multi-center cohort study with 9,156 patients. BMC Cancer 2013;13:487.

17. Wockel A, Kurzeder C, Geyer V, et al. Effects of guideline adherence in primary breast cancer-a 5-year multi-center cohort study of 3976 patients. Breast 2010;19:120-127.

18. Denu RA, Hampton JM, Currey A, et al. Influence of patient, physician, and hospital characteristics on the receipt of guideline-concordant care for inflammatory breast cancer. Cancer Epidemiol 2016;40:7-14.

19. Carrick S, Parker S, Wilcken N, et al. Single agent versus combination chemotherapy for metastatic breast cancer. Cochrane Database Syst Rev 2005:CD003372.

20. Carrick S, Parker S, Thornton CE, et al. Single agent versus combination chemotherapy for metastatic breast cancer. Cochrane Database Syst Rev 2009:CD003372.

21. Dear RF, McGeechan $K$, Jenkins $M C$, et al. Combination versus sequential single agent chemotherapy for metastatic breast cancer. Cochrane Database Syst Rev 2013:CD008792.

22. Treska V, Cerna M, Kydlicek T, Treskova I. Prognostic factors of breast cancer liver metastasis surgery. Arch Med Sci 2015;11:683-685.

23. New NCCN Guidelines include evidence blocks to illustrate value in breast, colon, kidney, and rectal cancers. J Natl Compr Canc Netw 2016; 14:xxxiv-xxxv.

24. Feinberg BA, Lang J, Grzegorczyk J, et al. Implementation of cancer clinical care pathways: a successful model of collaboration between payers and providers. J Oncol Pract 2012;8(3 Suppl):e38s-43s.

25. Kreys ED, Koeller JM. Documenting the benefits and cost savings of a large multistate cancer pathway program from a payer's perspective. J Oncol Pract 2013;9:e241-247.

See JNCCN.org for supplemental online content. 